\title{
The Effects of a Self-Care Program on Promoting Self-Care Behaviors in Patients with Psoriasis
}

\author{
Hojatolah Karimipour, ${ }^{1}$ Neda Sayadi, ${ }^{2}$ Abdolali Shariati, ${ }^{3, *}$ Mohammad Hossein Haghighi, ${ }^{4}$ and Reza \\ Yaghoubi ${ }^{5}$ \\ ${ }^{1}$ Student Research Committee, Ahvaz Jundishapur University of Medical Sciences, Ahvaz, Iran \\ ${ }^{2}$ Phd Candidate in Nursing, Faculty of Nursing Department, Ahvaz Jundishapur University of Medical Sciences, Ahvaz, IR Iran \\ ${ }^{3}$ Nursing Care Research Center in Chronic Diseases, Faculty of Nursing Department, Ahvaz Jundishapur University of Medical Sciences, Ahvaz, IR Iran \\ ${ }^{4}$ Master of Biostatistic Faculty of Public Health Department, Ahvaz Jundishapur University of Medical Sciences, Ahvaz, IR Iran \\ ${ }^{5}$ Subspecialist Dermatologist Professor, Faculty of Medicine Department, Ahvaz Jundishapur University of Medical Sciences, Ahvaz, IR Iran \\ "Corresponding author: Abdolali Shariati, Nursing Care Research Center in Chronic Diseases, Ahvaz Jundishapur University of Medical Sciences, Ahvaz, IR Iran. E-mail: \\ shariati-a@ajums.ac.ir
}

Received 2017 April 17; Revised 2017 May 29; Accepted 2017 July 04.

\begin{abstract}
Background: Psoriasis is a chronic inflammatory disease with frequent recurrence. The associated complications cause disturbance in patients' quality of life. In addition to treatment procedures, use of self-care behaviors can be considered a cost-effective and applicable method in improving the patients' symptoms. Therefore, the present study aimed to investigate the effectiveness of a self-care program in promoting self-care behaviors of patients with psoriasis.

Objectives: The present study aimed to investigate the effectiveness of a self-care program in promoting self-care behaviors among patients with psoriasis.

Methods: This quasi experimental, before-after study was carried out on 32 patients. The educational program was implemented face-to-face and in groups (in areas such as medicine, bathing, and clothing). The samples were examined once every 2 weeks for 3 months. Two questionnaires and 1 self-report checklist with a correlation coefficient of $92.5 \%$ were used in this study. The validity of the questionnaires was determined using content validity. Data were analyzed using repeated measures ANOVA in SPSS.

Results: The patients' demographics were as follows: mean age, 42.37\%; males, 16 (55.2\%) and females, 13 (44.8\%); married, 21 (72.4\%), single, 6 (20.7\%), and widowed, 2 (6.9\%); secondary school education, 13 (44.8\%); average disease period, 9.03\%; most self-employed and housewives (9, 31\%); history of disease, 7 (24.1\%); and physician-diagnosed disease, 29 (100\%). The results showed that the mean medication adherence rate increased from $7 \%$ to $9.72 \%$ after the intervention, and comparison of self-care behaviors before and after the intervention showed that the frequency of most behaviors significantly increased.

Conclusions: The results showed that training could improve self-care behaviors. Use of simple, low-cost, and available programs for self-care behaviors is effective in reducing the patients' symptoms and improving their conditions.
\end{abstract}

Keywords: Psoriasis, Self-Care, Education

\section{Background}

Psoriasis is a chronic genetic inflammatory hypoproliferative disease with flaked layers (1-4). The exact cause of this disease is not fully understood yet (5). It is equally prevalent in men and women (1), affecting $1 \%-3 \%$ of the population worldwide (6), $3 \%$ of the American population (2), and $9 \%$ of the Iranians population (7). Moderate to severe psoriasis is associated with diseases, such as cardiovascular diseases, metabolic syndrome (1), anger, anxiety, frustration (5), increased risk of mortality (8), diabetes, hypertension, dyslipidemia (9), obesity (3), and reduced quality of life (10).

Moreover, $84 \%, 80 \%$, and $57 \%$ of patients suffer from pruritus, dry skin, and sleep disorders, respectively (11). Psoriasis accounts for $37 \%$ and $42 \%$ of skin irritations and pain complaints, respectively (12). Depression is also a common disorder in these patients (13). In a study by Ghorbani et al. in 2012 on 854 patients with psoriasis, referred to Imam Khomeini Hospital from 2006 to 2011, it was revealed that $36 \%$ of the patients had more than 1 psoriasis-related disorder.

Treatment of psoriasis is time-consuming and requires repeated visits to dermatologists (14). The aim of treatment is to reduce inflammation and skin cell division (15). Psoriasis is a costly disease, which imposes major costs on patients and is significantly associated with the severity of disease (8). It also imposes a substantial health economic burden (16); in fact, the cost of treatment for this disease is $70 \%$ higher than that of similar diseases (17).

Although psoriasis can be controlled pharmaceuti- 
cally, great costs are imposed on patients, while other nonpharmaceutical methods, such as training, can be more cost-effective (11). Therefore, health education for patients is of great importance (18). Self-management education is the cornerstone of treatment (19), and awareness of disease and understanding its severity are very effective in alleviating the patients' pain and suffering (6).

Education promotes the individual's awareness of the disease, increases his/her motivation to make behavioral changes, and subsequently improves the clinical outcomes (19). On the other hand, training self-care behaviors for patients can have major effects on promoting the patients' health status and quality of life (20). Moreover, it can increase the patients' feeling of control over the disease and result in better skin care procedures (21).

Although self-care behaviors play a major role in the control of chronic diseases (22), level of such behaviors is not satisfactory among patients, and many treatments have minor positive effects, besides different side effects and long response time, leading to an increase in recurrence and prolonged treatment course (12). Therefore, it is important to train self-care behaviors and establish selfmanagement interventions for patients (19).

Few studies have been conducted in the field of selfcare among patients with psoriasis. Only 1 similar study has been performed in Iran, while no foreign research was found, despite several searches in the literature. Since training and implementation of self-care behaviors are effective in improving psoriasis, the present study aimed to determine the effects of self-care behaviors on the improvement of self-care in patients with psoriasis.

\section{Methods}

This quasi experimental study was carried out on 32 patients with psoriasis, who were referred to a dermatology clinic and Imam Khomeini public teaching hospital in Ahvaz, Iran (with 6 dermatologists and 22 active beds), using convenience sampling. Considering a 10\% loss to followup, $99 \%$ confidence interval, and $95 \%$ statistical power for the self-score difference before and after training, the following equation was used:

$n=\frac{\left(Z 1-\frac{\propto}{2}+Z 1-\beta\right)^{2} \times S d^{2}}{\bar{d}^{2}}=29$

During the intervention, 3 patients were excluded, while 29 participants remained in the study. For sampling, the researcher visited the center every day (from Saturday to Wednesday) and interviewed the patients. Participation of patients was based on their consent. Before completing the questionnaire, the study objectives were explained to the participants.
The disease was diagnosed by a dermatologist, and subjects were enrolled in the study, based on the inclusion criteria: 1) at least a 1-year history of psoriasis; 2) age above 15 years; 3) ability to understand and speak Farsi; and 4) no history of formal education in self-care. On the other hand, the exclusion criteria were as follows: 1) lack of full response to the questionnaire; 2) nonparticipation in a training session; and 3) noncompliance with self-care behaviors.

The intervention was carried out in the field of skin care and drug regimen, and pre- and postintervention information about self-care behaviors was collected and compared. Data were collected using 2 questionnaires and 1 checklist. The questionnaires included demographic characteristics (marital status, educational level, duration of disease, family history of disease and other autoimmune diseases, and sources of information about the disease). Questionnaires assessing self-care behaviors (28 multiple-choice questions to assess patients' self-care behaviors regarding skin care and treatment regimens) were completed by interviewing the patients.

Self-care was scored as follows: always (3 points), sometimes or self-care less than 4 days a week ( 2 points), seldom or less than 2 days a week ( 1 point), and never ( 0 point). The total score was obtained from the sum of responses, with higher scores indicating better self-care. The minimum score was 28 and the maximum score was 84. For assessing the reliability and content validity of the self-care need-assessment questionnaire (researchermade), the viewpoints of 10 faculty members of Ahvaz Nursing University were collected. The desired changes were applied, and a pilot study was conducted on 15 samples, which showed that all questions were applicable in 1 schema; its reliability was confirmed with a Cronbach's alpha of $0.82(\mathrm{P}<0.001)$.

The self-care self-report checklist (1 question on drug regimen and 5 questions on skin care) was also used to determine skin care, type and dose of medications, bathing frequency, type of household soap, water temperature, skin cleansing manner, body wash, and dress material. Scoring was based on the comparison of mean self-care behaviors before and after the intervention. Content validity and concurrent reliability were used to respectively determine the validity and reliability of data collection tools (self-care checklist). A reliability correlation coefficient of 92.5\% was obtained for the checklist (11).

The checklists were completed twice within 2 weeks before the intervention (no intervention was performed by the researcher at this stage) and 2 weeks later during 3 months after the intervention. The Orem's general theory was used for training, as it is based on self-care in nursing and includes activities that individuals use in order 
to maintain, restore, or improve health on their own (23). Self-care behaviors were trained to patients in 2 one-and-ahalf-hour face-to-face individual and group sessions (n, 7); the training manual was given to them at the end of the sessions.

Meetings started with the introduction of members to each other and explanation of group work and duration of sessions. The discussed topics included current problems in the treatment of psoriasis, complications of the disease, the patient's role in disease management, and education of self-care skin behaviors. In the second session, topics of the previous session and appropriate drug regimen were discussed with questions and answers, and a summary of issues was presented; trainings continued for 3 months. The drug regimen recall checklists were available to the patients every 2 weeks for 3 months (during the first 3 months) and were completed.

The researcher attended the dermatology clinic at the end of each month for better tracking of program implementation and completion of checklists. He also solved the patient's problems and followed the program both in person and through phone calls. After 3 months of training self-care behaviors and completion of self-care checklists after the end of each month, pre-and postintervention data were compared. Data were analyzed using repeated measures ANOVA in SPSS.

This study was registered by the ethics code, IR.AJUMS.IR.1394.553 (No., u-94171 on November 21, 2015). The ethical considerations in this study included a formal introduction letter from the department of research for presentation to Imam Khomeini hospital of Ahvaz. Before the intervention, the scope and nature of the study were explained to all the subjects. To participate in the study, informed consents were obtained from the subjects, and they were free to enter or leave the study whenever they desired. They were also assured that their information would remain confidential and only investigated generally.

\section{Results}

The analyses in this study, as presented in Table 1, showed the following results in the studied patients: $55.2 \%$ male; $42.37 \%$ at mean age; $31 \%$ housekeepers; $72.4 \%$ married; $24.1 \%$ with education above high school diploma; average disease period of 9.3 years; history of disease in $75.9 \%$; and physician diagnosis rate of $100 \%$. Also, according to the comparison of self-care behaviors before and after the intervention, as presented in Table 2, it can be concluded that most of these behaviors significantly increased in frequency.

Preintervention medication adherence was $7 \%$, which reached to $9.72 \%$ after the intervention, thus indicating
Table 1. The Frequencies and Percentage Distribution of the Demographic Characteristics of the Subjects

\begin{tabular}{|c|c|c|}
\hline & Frequency & Percentage \\
\hline \multicolumn{3}{|l|}{ Gender } \\
\hline Male & 16 & 55.2 \\
\hline Female & 13 & 44.8 \\
\hline \multicolumn{3}{|l|}{ Occupation } \\
\hline Worker & 1 & 3.4 \\
\hline Employee & 7 & 24.1 \\
\hline Housewife & 9 & 31 \\
\hline Self-employed & 9 & 31 \\
\hline Student & 2 & 6.9 \\
\hline Secretary & 1 & 3.4 \\
\hline \multicolumn{3}{|l|}{ Marital status } \\
\hline Married & 21 & 72.4 \\
\hline Single & 6 & 20.7 \\
\hline Widow & 2 & 6.9 \\
\hline \multicolumn{3}{|l|}{ Level of education } \\
\hline Illiterate & 1 & 3.4 \\
\hline Primary school & 2 & 6.9 \\
\hline Middle school & 6 & 20.7 \\
\hline High school diploma & 13 & 44.8 \\
\hline Above high school diploma & 7 & 24.1 \\
\hline \multicolumn{3}{|l|}{ Source of information } \\
\hline Doctor & 29 & 100 \\
\hline Nurse & 0 & 0 \\
\hline Family & 0 & 0 \\
\hline Medical journals & 0 & 0 \\
\hline Media & 0 & 0 \\
\hline \multicolumn{3}{|l|}{ History of disease } \\
\hline Yes & 7 & 24.1 \\
\hline No & 22 & 75.9 \\
\hline \multicolumn{3}{|l|}{ other diseases } \\
\hline Yes & 2 & 6.9 \\
\hline No & 27 & 93.1 \\
\hline
\end{tabular}

a significant difference. On the other hand, the average skin care behaviors, including use of cotton dress, bathing, and water temperature, were $4.86 \%, 4.89 \%$, and $3.48 \%$, respectively before the intervention, which changed to $8.58 \%$, $6.27 \%$, and $4.27 \%$ after the intervention, respectively; the results indicated a significant difference. However, the average skin care behaviors in use of soft washcloth and neutral 
Table 2. Comparison of the Mean Self-Care Behaviors 2 Weeks Before and 2 Weeks After the Program

\begin{tabular}{|c|c|c|c|c|c|c|}
\hline \multirow[t]{2}{*}{ Self-Care Behaviors } & \multicolumn{3}{|c|}{ Two Weeks Before the Program } & \multicolumn{3}{|c|}{ Two Weeks After the Program } \\
\hline & Mean & Standard Deviation & PValue & Mean & Standard Deviation & PValue \\
\hline Medicine consumption & 7.0000 & 2.10442 & $\mathrm{P}<0.001$ & 9.7241 & 0.64899 & $\mathrm{P}<0.001$ \\
\hline Soft sponges & 5.4483 & 2.68043 & 0.333 & 6.2069 & 0.90156 & 0.148 \\
\hline Neutral soap & 6.7586 & 2.50222 & 0.138 & 7.4138 & 1.29607 & 0.092 \\
\hline Cotton clothes & 4.8621 & 3.58294 & $\mathrm{P}<0.001$ & 8.5862 & 1.29607 & $\mathrm{P}<0.001$ \\
\hline Bathing & 4.897 & 3.59906 & 0.017 & 6.276 & 0.88223 & 0.018 \\
\hline Water temperature & 3.4828 & 2.66754 & 0.014 & 4.2759 & 1.13063 & 0.036 \\
\hline
\end{tabular}

soap were $5.44 \%$ and $6.75 \%$ and $6.20 \%$ and $7.41 \%$ before and after the intervention, respectively, and the difference was not significant.

\section{Discussion}

Researchers have used several methods and different drugs to improve the conditions of psoriatic patients; however, no definite treatment has been found yet for this disease. There has been a major focus on drug treatments, while less attention has been paid to the role of education. The present study aimed to determine the effects of selfcare behaviors on the improvement of self-care in patients with psoriasis.

Self-care program training can be accepted and implemented at home by patients, considering its costeffectiveness, understandability, and applicability. The results showed that the mean age of the patients was 42.37 years in this study, which is in line with a study by Ansar and colleagues (24). Many patients were male in our study, which is in accordance with a study by Ghorbani et al.; this finding may be attributed to the higher exposure of men to environmental, physical, and psychological stress (14).

The average medication adherence was $7 \%$ and 9.72\% before and after the intervention, respectively, which shows a significant difference. In line with our study, the medication adherence rate ranged from $33 \%$ to $78 \%$ in a study by Gorin et al., and one-third of the patients discontinued their regular treatment due to forgetfulness to use their medicine, high cost of drugs, long duration of medical treatment, and other medical problems (25). On the other hand, Joeteck et al. reported a medication adherence rate of $56.5 \%$, which is consistent with the present study (26).

Saeki et al. showed an average ointment use of $4.3 \%$. Previous studies have shown that adherence to oral medications is more than topical medications and is attributed to the higher age and annual income of patients (27). In addition, Brown et al. reported a medication adherence rate of 57\%, which is consistent with the present study (28). Therefore, timely and appropriate psoriasis treatment can control the disease, prevent further complications, and reduce the overall cost imposed on the economy of the community.

In addition, psoriasis, the associated diseases, and their complications reduce the quality of life and impose a huge economic burden on patients and society due to the increased frequency of hospitalization, loss of productivity, and absenteeism (26). Skin self-care behavioral patterns changed from $4.86 \%$ to $8.58 \%$, from $4.89 \%$ to $6.27 \%$, and from $3.48 \%$ to $4.27 \%$, respectively in terms of cotton clothing, bathing, and water temperature, which demonstrates the effectiveness of education; this finding is consistent with a study conducted by Mansouri et al. (11).

However, it should be noted that training was not effective in case of using soft washcloth and neutral soap. The patterns of soft washcloth and mild soap use changed from $5.44 \%$ to $6.20 \%$ and from $6.75 \%$ to $7.41 \%$, respectively, which may be due to severe itching while showering. Itching occurs due to reasons such as use of stimulating and fragrant soaps, which cause skin dryness, irritation, and itching and lead to the patients' use of rough washcloths to reduce skin itching. Therefore, it results in more skin irritation and redness, and subsequent recovery is delayed.

Psoriasis is one of the most costly chronic skin diseases (29). Self-care behaviors play the most important role in the control of chronic diseases (22), and self-care programs have a significant effect on improving and controlling diseases. The treatment costs are quite affordable for patients, since the cost of treatment is 70\% higher than that of similar diseases (17). Also, admission costs, outpatient treatment costs, and total annual cost of healthcare are about 1056, 2151, and 18960 dollars per patient, respectively.

The total direct costs associated with psoriasis were estimated at 5 million dollars in the United States in 2006 (13); therefore, the cost of relapse and recurrence can be 
reduced from 7706 to 25 Euros by implementing nonpharmacological methods (8). Patient education is one of the best nonpharmacological methods and one of the most important roles of nurses (30). Effective training is patientcentered, takes the patient's specific training needs into account (31), and uses functional, simple, and low-cost tools.

\subsection{Conclusion}

The results of the present study showed that the majority of the patients had moderate self-care behaviors, and some required behavioral modifications. Therefore, for program planning, implementation, and following selfcare training programs in psoriasis, authorities should pay attention to targeted self-care educational programs and regular repetition of the programs; also, after the primary education, self-care practices should be followed in patients for regular compliance with the program. With regard to the recovery of symptoms, changes in the application of self-care behaviors are made after raising the patients' awareness of disease condition and their understanding of the care concept of their participation. Therefore, this solution can be used as an understandable, lowcost, and applicable method by patients to overcome the disease and prevent its recurrence. According to the results and the effects of education, educational packages for patients and nurses are suggested as an important step to improve the patients' status.

The present study had some limitations. First, it was not performed on 2 case and control groups. Second, individual differences and motivations of the participants, which might affect the learning process and the results, were out of the researcher's control. As few studies have been performed in the field of self-care behaviors for psoriasis, the present study can motivate future research in this field.

\section{Footnote}

Conflicts of Interest: The authors declare no conflicts of interest.

\section{References}

1. Cordiali-Fei P, Bianchi L, Bonifati C, Trento E, Ruzzetti M, Francesconi F. Immunologic biomarkers for clinical and therapeutic management of psoriasis. Hindawi Publish Cor Med Inflam. 2014.

2. Hagg D, Eriksson M, Sundstrom A, Schmitt-Egenolf M. The higher proportion of men with psoriasis treated with biologics may be explained by more severe disease in men. PLoS One. 2013;8(5):e63619. doi: 10.1371/journal.pone.0063619. [PubMed: 23691076].

3. Hsu L, Armstrong AW. Treatment efficacy and safety profile in patients with psoriasis. Immunol Res. 2014.

4. Puig L, Fan T, Ding Q, Smith NE. Predictors of biologic treatment of psoriasis: a non-interventional study. Clinicoecon Outcomes Res. 2014;6:93-100. doi: 10.2147/CEOR.S54797. [PubMed: 24600238].
5. Doukaki S, Caputo V, Bongiorno MR. Psoriasis and Cardiovascular Risk: Assessment by CUORE Project Risk Score in Italian Patients. Dermatol Res Pract. 2013;2013:389031. doi: 10.1155/2013/389031. [PubMed: 24082881].

6. Augustin M, Alvaro-Gracia JM, Bagot M, Hillmann O, van de Kerkhof PC, Kobelt G, et al. A framework for improving the quality of care for people with psoriasis. J Eur Acad Dermatol Venereol. 2012;26 Suppl 4:116. doi: 10.1111/j.1468-3083.2012.04576.x. [PubMed: 22725729].

7. Jamshidi F, Bouzari N, Seirafi H, Farnaghi F, Firooz A. The prevalence of psoriatic arthritis in psoriatic patients in Tehran, Iran. Arch Iran Med. 2008;11(2):162-5. [PubMed: 18298293].

8. Mustonen A, Leino M, Mattila K, Koulu L, Tuominen R. Treatment costs of psoriasis in a tertiary-level clinic. BMC Health Serv Res. 2014;14.

9. Ahlehoff O, Lindhardsen J, Gislason GH, Olesen JB, Charlot M, Skov $\mathrm{L}$, et al. Prognosis after percutaneous coronary intervention in patients with psoriasis: a cohort study using Danish nationwide registries. BMC Cardiovasc Disord. 2012;12:79. doi: 10.1186/1471-2261-12-79. [PubMed: 23006590].

10. Cawson MR, Mitchell SA, Knight C, Wildey H, Spurden D, Bird A, et al. Systematic review, network meta-analysis and economic evaluation of biological therapy for the management of active psoriatic arthritis. BMC Musculoskelet Disord. 2014;15:26. doi: 10.1186/1471-2474-15-26. [PubMed: 24444034].

11. Mansouri M, Navipour H, Memarian R. The effect of self-care program on relief of skin complications of psoriasis. ZUMSJ. 2006;14(54):24-31.

12. Augustin M, Radtke MA. Quality of life in psoriasis patients. Expert Rev Pharmacoecon Outcomes Res. 2014;14(4):559-68. doi: 10.1586/14737167.2014.914437. [PubMed: 24820452].

13. Feldman SR, Zhao Y, Shi L, Tran MH, Lu J. Economic and comorbidity burden among moderate-to-severe psoriasis patients with comorbid psoriatic arthritis. Arthritis Care Res (Hoboken). 2015;67(5):708-17. doi: 10.1002/acr.22492. [PubMed: 25303478].

14. Alireza GB, Ali F, Ashrafsadat H. Epidemiologic study of psoriasis and concomitant diseases among patients referred to dermatology clinic of Imam Khomeini hospital, Ahvaz 2006 to 2011. Jundishapur J Chronic Disease Care. 2015;1(1):1-9.

15. Griffiths C, Barker J, Bleiket T, Chalmers RDC. Rook's textbook of dermatology. 9ed ed. Blackwell Scince; 2016.

16. Steinke SI, Peitsch WK, Ludwig A, Goebeler M. Cost-of-illness in psoriasis: comparing inpatient and outpatient therapy. PLoS One. 2013;8(10):e78152. doi: 10.1371/journal.pone.0078152. [PubMed: 24194911].

17. Crown WH, Bresnahan BW, Orsini LS, Kennedy S, Leonardi C. The burden of illness associated with psoriasis: cost of treatment with systemic therapy and phototherapy in the US. Curr Med Res Opin. 2004;20(12):1929-36. doi: 10.1185/030079904X15192. [PubMed: 15701211].

18. Jankowiak B, Krajewska-Kulak E, Van Damme-Ostapowicz K, Wronska I, Lukaszuk C, Niczyporuk W, et al. The need for health education among patients with psoriasis. Dermatol Nurs. 2004;16(5):43944. [PubMed: 15624709].

19. Larsen MH, Hagen KB, Krogstad AL, Aas E, Wahl AK. Limited evidence of the effects of patient education and self-management interventions in psoriasis patients: a systematic review. Patient Educ Couns. 2014;94(2):158-69. doi: 10.1016/j.pec.2013.10.005. [PubMed: 24184041].

20. Rahimi A, Salehi S, Afrasiabifar A. The effect of orem's self-care model on quality of life of patients with hypothyroid goiter. Armaghane danesh. 2012;17(5):0.

21. Skarpathiotakis M, Fairlie C, Ryan S. Specialized education for patients with psoriasis: a patient survey on its value and effectiveness. Dermatol Nurs Asso. 2006;18(4):358.

22. Parham M, Rasooli A, Safaeipour RSM. Assessment of effects ofself caring on diabetic patients in qom diabetes. J Sabzevar Unive Med Sci. 2014:84-473. 
23. Masoudi R, Mohammadi I, Ahmadi F, Hasanpour-Dehkordi A. The effect of self-care program education based on Orem'stheory on mental aspect of quality of life in multiple sclerosis patients. Iran J Nurs. 2009;22(60):53-64.

24. Ansar A, Jahangard L, Pahlevani P, Rasouli B, Torabian S, Rasouli S. Quality of life in patients with psoriasis vulgaris: A case-control study. Dermatol Cosmetic. 2013;4(3).

25. Goren A, Carter C, Lee S. Patient reported health outcomes and non-adherence in psoriasis patients receiving adalimumab or ustekinumab for moderate to severe plaque psoriasis. $J$ Dermatolog Treat. 2016;27(1):43-50. doi: 10.3109/09546634.2015.1049972. [PubMed: 26088404].

26. Jevtic T, Bukumiric Z, Jankovic SM. Effects of treatment adherence on clinical and economic outcomes in patients with psoriasis. Med Glas (Zenica). 2013;10(1):106-12. [PubMed: 23348171].

27. Saeki H, Imafuku S, Abe M, Shintani Y, Onozuka D, Hagihara A, et al. Poor adherence to medication as assessed by the Morisky Medication Adherence Scale- 8 and low satisfaction with treatment in 237 psoriasis patients. J Dermatol. 2015;42(4):367-72. doi: 10.1111/1346-8138.12804.
[PubMed: 25720544]

28. Brown KK, Rehmus WE, Kimball AB. Determining the relative importance of patient motivations for nonadherence to topical corticosteroid therapy in psoriasis. J Am Acad Dermatol. 2006;55(4):607-13. doi: 10.1016/j.jaad.2005.12.021. [PubMed: 17010739].

29. Aliasgharpour M, Shomali M, Moghaddam MZ, Faghihzadeh S. Effect of a self-efficacy promotion training programme on the body weight changes in patients undergoing haemodialysis. $J$ Ren Care. 2012;38(3):155-61. doi: 10.1111/j.1755-6686.2012.00305.x. [PubMed: 22429325].

30. Dragoi RG, Ndosi M, Sadlonova M, Hill J, Duer M, Graninger W, et al. Patient education, disease activity and physical function: can we be more targeted? A cross sectional study among people with rheumatoid arthritis, psoriatic arthritis and hand osteoarthritis. Arthritis Res Ther. 2013;15(5):R156. doi: 10.1186/ar4339. [PubMed: 24286444].

31. Kalantari S, Najafi K, Abbaszadeh A, Sanagoo A, Borhani F. Nurses' perception of performance of patient education. JentashapirJ Health Res. 2012;2(4):167-74. 